\title{
Multiwavelength studies of the Seyfert 1 galaxy NGC 7469 I. Far UV observations with FUSE
}

\author{
G. A. Kriss ${ }^{1,2}$, A. Blustin ${ }^{3}$, G. Branduardi-Raymont ${ }^{3}$, R. F. Green ${ }^{4}$, J. Hutchings ${ }^{5}$, and M. E. Kaiser ${ }^{2}$ \\ 1 Space Telescope Science Institute, 3700 San Martin Drive, Baltimore, MD 21218, USA \\ e-mail: gak@stsci.edu \\ 2 Center for Astrophysical Sciences, Johns Hopkins University, Baltimore, MD 21218-2686, USA \\ 3 Mullard Space Science Laboratory, University College London, Holmbury St. Mary, Dorking, Surrey, RH5 6NT, UK \\ ${ }^{4}$ Kitt Peak National Observatory, National Optical Astronomy Observatories, PO Box 26732, 950 North Cherry Ave., Tucson, \\ AZ 85726-6732, USA \\ e-mail: rgreen@noao.edu \\ ${ }^{5}$ Dominion Astrophysical Observatory, National Research Council of Canada, Victoria, BC, V8X 4M6, Canada \\ e-mail: john.hutchings@hia.nrc.ca
}

Received 23 December 2002 / Accepted 14 February 2003

\begin{abstract}
We obtained far-ultraviolet spectra of the Seyfert 1 galaxy NGC 7469 using the Far Ultraviolet Spectroscopic Explorer on 1999 December 6. Our spectra cover the wavelength range 990-1187 $\AA$ with a resolution of $\sim 0.05 \AA$. We see broad emission lines of C III, N III, O VI, and He II as well as intrinsic absorption lines in the O VI $\lambda \lambda 1032,1038$ resonance doublet. The absorption arises in two distinct kinematic components at systemic velocities of $-569 \mathrm{~km} \mathrm{~s}^{-1}$ and $-1898 \mathrm{~km} \mathrm{~s}^{-1}$. Both components are very highly ionized - no significant $\operatorname{Ly} \beta$ absorption is present. The higher blueshift component is not quite saturated, and it has a total O VI column density of $8 \times 10^{14} \mathrm{~cm}^{-2}$. It covers more than $90 \%$ of the continuum and broadline emission. The lower blueshift component is heavily saturated and covers only $\sim 50 \%$ of the continuum and broad-line emission. It too has a column density of $8 \times 10^{14} \mathrm{~cm}^{-2}$, but this is less certain due to the high saturation. We set an upper limit of $<1.5 \times 10^{18} \mathrm{~cm}^{-2}$ on the O VI column density of this component. Its line depth is consistent with coverage of only the continuum, and thus this component may lie interior to the broad emission-line gas. The component at $-569 \mathrm{~km} \mathrm{~s}^{-1}$ has a velocity comparable to the high-ionization X-ray absorption lines seen in the XMM-Newton grating spectrum of NGC 7469, and photoionization models show that the observed column densities of O VI and H I are compatible with their formation in the same gas as that causing the X-ray absorption. The gas at $-1898 \mathrm{~km} \mathrm{~s}^{-1}$ has lower ionization and column density, and no significant $\mathrm{X}$-ray absorption is associated with it.
\end{abstract}

Key words. galaxies: active - galaxies: individual (NGC 7469) - galaxies: quasars: absorption lines - galaxies: Seyfert ultraviolet: galaxies $-\mathrm{X}$-rays: galaxies

\section{Introduction}

The Seyfert 1 galaxy NGC 7469 is one of a handful of bright, nearby active galactic nuclei (AGN) that has been intensively studied at multiple wavelengths and monitored extensively in the X-ray, UV, and optical bands (Wanders et al. 1997; Nandra et al. 1998; Collier et al. 1998; Kriss et al. 2000a). ASCA X-ray spectra (Reynolds 1997; George et al. 1998) were modeled with absorption edges of O VII and O VIII indicative of a warm absorber, and Hubble Space Telescope Faint Object Spectrograph observations show associated UV absorption lines in Ly $\alpha$, $\mathrm{NV}$ and CIV. Such X-ray and UV absorption is common in Seyfert $1 \mathrm{~s}$, occurring in tandem in roughly half the population (Reynolds 1997; George et al. 1998; Crenshaw et al. 1999). The

Send offprint requests to: G. A. Kriss, e-mail: gak@stsci .edu Correspondence to: G. A. Kriss, Space Telescope Science Institute, 3700 San Martin Drive, Baltimore, MD 21218, USA.
X-ray and UV absorbing gas components thus appear to be related, but it is not yet clear whether this is the consequence of a more general phenomenon linking the gas visible at both wavelengths, or if the absorption arises in the same gas with identical physical conditions in both the X-ray and the UV. At high resolution, both the X-ray and the UV absorption is kinematically complex (Kaastra et al. 2001; Kaspi et al. 2000; Kaspi et al. 2002; Crenshaw et al. 1998; Crenshaw et al. 1999; Mathur et al. 1999; Kriss et al. 2000b). Generally only some of the UV absorption lines have velocities and physical conditions similar to those seen in the X-ray.

The locations of both the X-ray and the UV absorbing media are also highly uncertain. If the outflowing gas originates in the accretion disk, then it may be relatively close to the central engine (e.g., Königl \& Kartje 1994; Murray et al. 1995) and interior to the broad emission-line region. On the other hand, if the absorbing gas is a thermal wind driven off the obscuring 
torus (Krolik \& Kriss 1995; Krolik \& Kriss 2001), then it will lie at distances of roughly $1 \mathrm{pc}$, be external to the broad-line region, but probably interior to the narrow-line region. Studies of the X-ray absorption variability (NGC 3516; Netzer et al. 2002) show that at least some of the gas does lie at distances approaching 1 pc. Variability of UV absorption in NGC 4151 suggests distances of tens of parsecs for the absorbing gas (Espey et al. 1998), and even greater distances for the associated UV absorption seen in some quasars (Hamann et al. 1995; Hamann et al. 1997).

Using the Far Ultraviolet Spectroscopic Explorer (FUSE), we obtained high-resolution UV spectra below $1200 \AA$ covering the $\mathrm{O}$ VI $\lambda \lambda 1032,1038$ resonance doublet and the highorder Lyman lines. The $\mathrm{O}$ VI doublet provides a key link to the $\mathrm{X}$-ray band since high energy transitions from the same ion can be viewed with XMM-Newton. This permits us to directly compare the kinematics and the column densities measured in both wavelength regions as a crucial test for establishing the link between X-ray and UV absorbing gas in Seyferts. XMM-Newton observations of NGC 7469 are discussed in a companion paper (Blustin et al. 2003).

\section{Observations}

FUSE observes the far-ultraviolet wavelength range from 912-1187 ̊ using four independent optical channels. In each channel a primary mirror gathers light for a Rowland-circle spectrograph. Two-dimensional photoncounting detectors record the dispersed spectra. Two of the optical trains use LiF coatings on the optics to cover the 990-1187 A wavelength range. The other two channels cover shorter wavelengths down to $912 \AA$ using SiC-coated optics. See Moos et al. (2000) for a full description of FUSE and its in-flight performance.

We observed NGC 7469 with FUSE on 1999 December 6 through the $30^{\prime \prime}$-square low-resolution apertures. Data were obtained on 22 consecutive orbits for a total integration time of 37803 s. Unfortunately the SiC channels were not properly aligned; the only detectable flux was in the LiF channels. The time-tagged data were processed using v1.8.7 of the FUSE calibration pipeline. Sahnow et al. (2000) describe the standard FUSE data processing steps. We added an additional customized step to normalize and subtract a flat background image from each detector segment. The resulting extracted spectra were merged into a linearized spectrum with $0.05 \AA$ bins. While this rebinning process introduces some correlated error, since each of these bins holds $\sim 7$ original pixels, the resulting bins are more than $80 \%$ statistically independent. Poisson errors and data quality flags are propagated through the data reduction process along with the science data.

To firmly establish the zero-point of our wavelength scale, we compare the positions of low-ionization Galactic absorption lines from species such as $\mathrm{Ar}$ I, Fe II, O I and $\mathrm{H}_{2}$ to the Galactic $21 \mathrm{~cm} \mathrm{H}$ I velocity as measured by Murphy et al. (unpublished). This requires a shift of $-0.32 \AA$ to be applied to our wavelengths to place them in a heliocentric reference frame. We estimate that the flux scale is accurate to $\sim 10 \%$, and that wavelengths are accurate to $\sim 15 \mathrm{~km} \mathrm{~s}^{-1}$.
The full merged spectrum is shown in the top panel of Fig. 1. Numerous Galactic absorption features obscure much of the spectrum, so we have constructed a simple model of the interstellar absorption to remove them from the spectrum. We assume a Galactic H I column of $4.4 \times 10^{20} \mathrm{~cm}^{-2}$ at a heliocentric velocity of $-9 \mathrm{~km} \mathrm{~s}^{-1}$ with a Doppler width of $10 \mathrm{~km} \mathrm{~s}^{-1}$. Heavy elements are included at solar abundances following the depletion pattern typical of warm gas toward $\zeta$ Oph (Savage $\&$ Sembach 1996). Matching the molecular hydrogen absorption requires one component with gas at $50 \mathrm{~K}$ and a $\mathrm{H}_{2}$ column density of $1 \times 10^{20} \mathrm{~cm}^{-2}$, and a second component with a temperature of $300 \mathrm{~K}$ and a total column density of $1 \times 10^{17} \mathrm{~cm}^{-2}$. We show the spectrum of NGC 7469 corrected for this foreground absorption in the lower panel of Fig. 1. Strong, broad $\mathrm{O}$ VI emission dominates the spectrum. Weaker broad emission lines of C III $\lambda 977$, N III $\lambda 991$, and He II $\lambda 1085$ are also visible, as well as several unidentified emission features. The low broad bump on the red wing of O VI is also present in the FUSE spectrum of Mrk 509 (Kriss et al. 2000b) as well as in the spectra of low-redshift quasars observed using the Hubble Space Telescope (Laor et al. 1995). Based on FUSE observations of a large sample of low-redshift AGN, Kriss et al. (2003) have identified this as blended emission due to S IV $\lambda \lambda 1062,1072$.

At the high resolution of FUSE, absorption features intrinsic to NGC 7469 are also discernible. Figure 2 shows the Ly $\beta$ and $\mathrm{O}$ VI region of the spectrum in $0.05 \AA$ bins. Here one can see two absorption systems due to the $\mathrm{O}$ VI $\lambda \lambda 1032,1038$ resonance doublet in the blue wing of the broad O VI emission line. The $\operatorname{Ly} \beta$ absorption associated with these systems is weak, if present at all, and severely blended with foreground Galactic absorption. Similarly, no Ly $\gamma$ or C III $\lambda 977$ absorption is visible at shorter wavelengths.

\section{Analysis}

To measure the emission and absorption line properties of NGC 7469, we model the spectrum using the IRAF task specfit (Kriss 1994). We use a reddened power law in $f_{\lambda}$ to describe the continuum emission. For extinction, we use a Cardelli et al. (1989) curve with $R_{V}=3.1$ and $E(B-V)=$ 0.069 (Schlegel et al. 1998). To model the continuum, we use regions of the spectrum free of both emission and absorption lines covering the full observed wavelength range. The bestfit, extinction-corrected continuum has the form $f_{\lambda}=(1.34 \pm$ $0.02) \times 10^{-13}(\lambda / 1000 \AA)^{-1.25 \pm 0.10} \mathrm{ergs} \mathrm{s}^{-1} \mathrm{~cm}^{-2} \AA^{-1}$.

To model the $\mathrm{O}$ VI emission and absorption, we fix the continuum parameters as determined above and restrict our subsequent fits to the 1033.0-1060.3 $\AA$ wavelength range. As one can see from the models shown in Fig. 2, the O VI emission line profile requires both broad and narrow components. We model these with Gaussian line profiles. The narrow component is only cleanly visible near the peak of the red component of the $\mathrm{O}$ VI doublet; strong foreground Galactic $\mathrm{H}_{2}$ absorption obscures the corresponding feature near the blue peak. Although we can only clearly see part of the red portion of the narrow doublet, our baseline model assumes that they are optically thin with a 2:1 intensity ratio. (Tests using other assumptions are described in our discussion of the absorption line fits below.) 


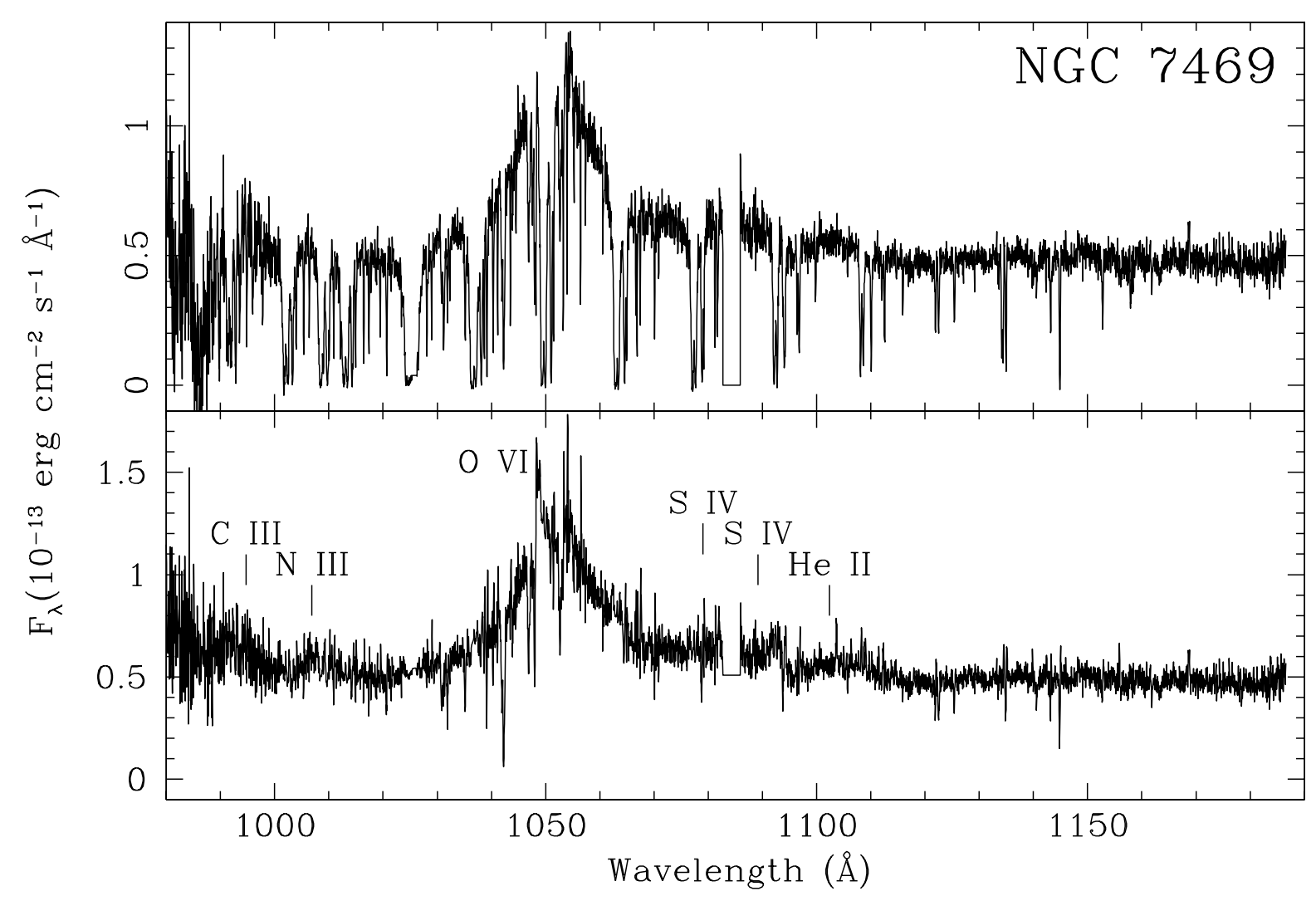

Fig. 1. Upper panel: observed FUSE spectrum of NGC 7469 with $0.05 \AA$ binning. Lower panel: the FUSE spectrum corrected for foreground interstellar absorption. Identified broad emission lines are marked.

The broad component is also treated as a doublet with two blended Gaussians having a 2:1 intensity ratio. For each narrow and broad doublet, we require the widths to be identical and the wavelengths to have the same ratio as their laboratory values. The C III $\lambda 977$, N III $\lambda 991$, S IV $\lambda \lambda 1062,1072$, and He II $\lambda 1085$ emission lines are all modeled as single Gaussian emission lines. The two components of the S IV doublet have identical widths, a fixed flux ratio of $1: 1$, and wavelengths fixed at the ratio of their laboratory value. In addition, we include a single Gaussian emission line at the location of the unidentified emission feature at $1031 \AA$. Table 1 gives the best-fit parameters for these emission lines.

We model absorption lines in the NGC 7469 spectrum using Voigt profiles. For the intrinsic absorbers, we permit the absorption to partially cover the source. That is, for a covering fraction $f_{\mathrm{c}}$, the transmittance at a given wavelength, $t(\lambda)$, has the form $t(\lambda)=1+f_{\mathrm{c}}\left(\mathrm{e}^{-\tau(\lambda)}-1\right)$, where $\tau(\lambda)$ is the optical depth at that wavelength. The intrinsic absorption lines are clustered into two complexes that we designate as \#1 (at a relative systemic velocity of $-569 \mathrm{~km} \mathrm{~s}^{-1}$ ) and \#2 (at velocity $-1898 \mathrm{~km} \mathrm{~s}^{-1}$ ). Each of these complexes is modeled with four separate blended absorption lines. The O VI lines in each component are treated as doublets with their wavelengths fixed at the ratio of their laboratory values, their optical depths fixed at a $2: 1$ ratio, and their velocity widths forced to be the same. For the corresponding $\operatorname{Ly} \beta$ lines, we fix their wavelengths at the ratio of their laboratory values to those of the $\mathrm{O}$ VI lines and force them to have the same velocity widths. Covering
Table 1. Emission lines in NGC 7469.

\begin{tabular}{|c|c|c|c|c|}
\hline Line & $\begin{array}{l}\lambda_{\text {vac }} \\
(\AA)\end{array}$ & Flux $^{a}$ & $\begin{array}{l}\text { Velocity } \\
\left(\mathrm{km} \mathrm{s}^{-1}\right)\end{array}$ & $\begin{array}{l}F W H M \\
\left(\mathrm{~km} \mathrm{~s}^{-1}\right)\end{array}$ \\
\hline C III & 977.02 & $9.5 \pm 2.1$ & $271 \pm 160$ & $1599 \pm 210$ \\
\hline $\mathrm{N}$ III & 991.58 & $5.3 \pm 1.3$ & $95 \pm 158$ & $1599 \pm 210$ \\
\hline Unknown & 1014.86 & $6.5 \pm 1.1$ & $0 \pm 160$ & $1939 \pm 518$ \\
\hline O vi broad & 1031.93 & $85.8 \pm 4.3$ & $323 \pm 37$ & $4901 \pm 146$ \\
\hline O vi broad & 1037.62 & $42.9 \pm 2.2$ & $323 \pm 37$ & $4901 \pm 146$ \\
\hline O VI narrow & 1031.93 & $23.5 \pm 2.9$ & $-163 \pm 55$ & $1061 \pm 82$ \\
\hline O VI narrow & 1037.62 & $11.8 \pm 1.5$ & $-163 \pm 55$ & $1061 \pm 82$ \\
\hline S IV & 1062.66 & $15.5 \pm 1.3$ & $194 \pm 142$ & $7637 \pm 251$ \\
\hline S IV & 1072.97 & $15.5 \pm 1.3$ & $194 \pm 142$ & $7637 \pm 251$ \\
\hline He II & 1085.15 & $3.2 \pm 0.3$ & $-47 \pm 161$ & $2575 \pm 210$ \\
\hline
\end{tabular}

a Observed flux in units of $10^{-14} \mathrm{erg} \mathrm{cm}^{-2} \mathrm{~s}^{-1}$.

b Velocity is relative to a systemic redshift of $c z=4916 \mathrm{~km} \mathrm{~s}^{-1}$ de Vaucouleurs et al. (1991).

fractions vary freely within each complex. The individual O VI lines within a complex are forced to have the same velocity widths and the same covering fractions. (This assumption is relaxed below in an alternative model for the absorption.) Since $\operatorname{Ly} \beta$ absorption is not even unambiguously detectable, we fix the relative optical depths of the lines within each complex to be the same as the ratios observed for O VI. However, we permit the overall optical depth for each $\operatorname{Ly} \beta$ complex to vary freely. Table 2 gives the best-fit parameters for this model of the absorption lines. 
Table 2. Absorption lines in NGC 7469.

\begin{tabular}{|c|c|c|c|c|c|c|c|}
\hline Feature & $\begin{array}{c}\text { Comp } \\
\#\end{array}$ & $\begin{array}{l}\lambda_{\text {vac }} \\
(\AA)\end{array}$ & $\begin{array}{l}W_{\lambda}^{\mathrm{a}} \\
(\AA)\end{array}$ & $\begin{array}{c}N_{\text {ion }} \\
\left(10^{12} \mathrm{~cm}^{-2}\right)\end{array}$ & $\begin{array}{c}\Delta v^{\mathrm{b}} \\
\left(\mathrm{km} \mathrm{s}^{-1}\right)\end{array}$ & $\begin{array}{l}F W H M \\
\left(\mathrm{~km} \mathrm{~s}^{-1}\right)\end{array}$ & $\overline{\overline{f_{\mathrm{c}}}}$ \\
\hline$\overline{\mathrm{Ly} \beta} \beta$ & $2 a$ & 1025.72 & $<0.05$ & $1.5 \pm 3.4$ & $-2016 \pm 12$ & $29 \pm 1$ & $0.93 \pm 0.03$ \\
\hline $\operatorname{Ly} \beta$ & $2 \mathrm{a}$ & 1025.72 & & $3.2 \pm 7.3$ & $-1975 \pm 4$ & $29 \pm 1$ & $0.93 \pm 0.03$ \\
\hline $\mathrm{Ly} \beta$ & $2 a$ & 1025.72 & & $12.9 \pm 29.5$ & $-1898 \pm 1$ & $29 \pm 1$ & $0.93 \pm 0.03$ \\
\hline $\operatorname{Ly} \beta$ & $2 \mathrm{a}$ & 1025.72 & & $4.0 \pm 9.1$ & $-1840 \pm 3$ & $29 \pm 1$ & $0.93 \pm 0.03$ \\
\hline $\mathrm{Ly} \beta$ & $1 \mathrm{a}$ & 1025.72 & $<0.03$ & $8.2 \pm 4.3$ & $-627 \pm 5$ & $25 \pm 2$ & $0.53 \pm 0.05$ \\
\hline $\mathrm{Ly} \beta$ & $1 b$ & 1025.72 & & $53.2 \pm 28.1$ & $-569 \pm 4$ & $25 \pm 2$ & $0.53 \pm 0.05$ \\
\hline $\operatorname{Ly} \beta$ & $1 \mathrm{c}$ & 1025.72 & & $15.3 \pm 8.1$ & $-506 \pm 3$ & $25 \pm 2$ & $0.53 \pm 0.05$ \\
\hline $\operatorname{Ly} \beta$ & $1 d$ & 1025.72 & & $3.9 \pm 2.1$ & $-431 \pm 5$ & $25 \pm 2$ & $0.53 \pm 0.05$ \\
\hline OVI & $2 \mathrm{a}$ & 1031.93 & $0.48 \pm 0.02$ & $36.1 \pm 16.2$ & $-2015 \pm 12$ & $29 \pm 1$ & $0.93 \pm 0.03$ \\
\hline OVI & $2 b$ & 1031.93 & & $121.2 \pm 16.3$ & $-1973 \pm 4$ & $29 \pm 1$ & $0.93 \pm 0.03$ \\
\hline OVI & $2 c$ & 1031.93 & & $494.0 \pm 51.4$ & $-1898 \pm 1$ & $29 \pm 1$ & $0.93 \pm 0.03$ \\
\hline OVI & $2 d$ & 1031.93 & & $154.4 \pm 16.9$ & $-1838 \pm 3$ & $29 \pm 1$ & $0.93 \pm 0.03$ \\
\hline OVI & 1a & 1031.93 & $0.23 \pm 0.02$ & $76.1 \pm 25.3$ & $-626 \pm 5$ & $25 \pm 2$ & $0.53 \pm 0.05$ \\
\hline OVI & $1 b$ & 1031.93 & & $494.7_{-167.2}^{+1.5 \times 10^{6}}$ & $-569 \pm 4$ & $25 \pm 2$ & $0.53 \pm 0.05$ \\
\hline OVI & $1 \mathrm{c}$ & 1031.93 & & $142.3 \pm 28.2$ & $-506 \pm 3$ & $25 \pm 2$ & $0.53 \pm 0.05$ \\
\hline OVI & $1 \mathrm{~d}$ & 1031.93 & & $58.7 \pm 15.0$ & $-432 \pm 5$ & $25 \pm 2$ & $0.53 \pm 0.05$ \\
\hline
\end{tabular}

${ }^{a}$ Equivalent widths are integrated over all components of each spectral feature. Upper limits are $2 \sigma$.

${ }^{\mathrm{b}}$ Velocity is relative to a systemic redshift of $c z=4916 \mathrm{~km} \mathrm{~s}^{-1}$ de Vaucouleurs et al. (1991).

Since much of the peak of the O VI emission-line profile is obscured by foreground Galactic absorption, we have explored the effects that this uncertainty in the true emission-line profile might have on our characterization of the absorption lines. We consider a wide range of models:

A. This is our baseline model, described in detail above. The narrow emission lines have line ratios fixed at an optically thin value of $2: 1$, and we assume that this narrow line emission is obscured at the same covering fraction as the continuum and broad emission line. Individual O VI lines within a complex have the same velocity widths and covering fractions.

B. In this model the narrow emission lines are treated the same as in "A", but the velocity widths and the covering fractions of individual $\mathrm{O}$ VI lines within a complex are all permitted to vary freely. In these fits, the strongest, deepest absorption line in each complex has parameters typical of those found in our baseline model. The weaker adjacent lines retain similar widths in these fits, but their best-fit covering fractions do change. However, the individual covering fractions are not well constrained. Their error bars are typically $\pm 20 \%$, and the new values lie within one error bar of the best-fit value in the baseline model.

C. This variation has the narrow emission line fluxes fixed at an optically thick ratio of $1: 1$. The emission is obscured with the same covering fraction as the continuum and broad emission line. As in the baseline model, individual $O$ VI lines within a complex have the same velocity widths and covering fractions. In a pattern that repeats with each of the subsequent variations below, the best-fit $\mathrm{O}$ VI column densities in this model are slightly lower, but not more than twice the values of the error bars shown in Table 2 . While this model significantly changes the overall emission-line profile, since the $\mathrm{O}$ VI absorption is so highly blueshifted, little of the intrinsic absorption falls in the region of the line profile with the most dramatic changes. Thus the overall optical depth of the absorption lines is roughly the same as in the baseline model.

D. We also considered a model with no narrow emission-line component. As in the baseline model, individual $\mathrm{O}$ VI lines within a complex have the same velocity widths and covering fractions. This model provides a significantly worse fit than the baseline, but the measured O VI column densities and covering fractions are the same as the baseline model to within the error bars in Table 2.

E. This model has narrow-line emission with line ratios fixed at 2:1, but here we assume that the intrinsic absorption does not obscure the narrow-line emission at all. As in the baseline model, individual O VI lines within a complex have the same velocity widths and covering fractions. Again the O VI column densities are lower, but they are not significantly different from those of the baseline model.

F. In this model we also assume that the intrinsic absorption does not absorb the narrow-line emission, but we fix that emission at a ratio of $1: 1$. As in the baseline model, individual $\mathrm{O}$ VI lines within a complex have the same velocity widths and covering fractions. Again we find no significant variation from the results of our baseline model.

The overall properties of these various emission-line profile models are summarized in Table 3 . For the $\mathrm{O}$ VI components 1 and 2 we list the total column density from the fit, the velocity of the strongest feature in each component, the best-fit widths of the absorption lines, and the best-fit covering fractions. The comparative summary for component \#1 is listed first in the table, followed by the summary for component \#2.

Figure 2 graphically illustrates our best fit to the O VI and $\operatorname{Ly} \beta$ region of the FUSE spectrum. Using our model for the underlying emission and the foreground Galactic absorption, we divide this into the data to produce a normalized spectrum that is corrected for the effects of foreground Galactic absorption. This normalized spectrum is shown in velocity space 
Table 3. Summary of properties of absorber models for NGC 7469.

\begin{tabular}{lcrccc}
\hline \hline Model/Component & $\chi^{2} / v$ & $\begin{array}{c}N_{\text {OVI }} \\
\left(10^{12} \mathrm{~cm}^{-2}\right)\end{array}$ & $\begin{array}{c}\Delta v^{\mathrm{a}} \\
\left(\mathrm{km} \mathrm{s}^{-1}\right)\end{array}$ & $\begin{array}{c}F W H M \\
\left(\mathrm{~km} \mathrm{~s}^{-1}\right)\end{array}$ & $f_{\mathrm{c}}$ \\
\hline $\mathrm{A} / 1$ & $604.0 / 344$ & $772 \pm 172$ & $-569 \pm 4$ & $25 \pm 2$ & $0.53 \pm 0.05$ \\
$\mathrm{~B} / 1$ & $596.9 / 332$ & $797 \pm 317$ & $-572 \pm 5$ & $24 \pm 6$ & $0.54 \pm 0.06$ \\
$\mathrm{C} / 1$ & $613.1 / 344$ & $820 \pm 217$ & $-569 \pm 4$ & $24 \pm 2$ & $0.51 \pm 0.04$ \\
$\mathrm{D} / 1$ & $747.5 / 344$ & $933 \pm 244$ & $-572 \pm 3$ & $24 \pm 2$ & $0.50 \pm 0.04$ \\
$\mathrm{E} / 1$ & $622.1 / 344$ & $758 \pm 163$ & $-569 \pm 3$ & $25 \pm 2$ & $0.56 \pm 0.05$ \\
$\mathrm{~F} / 1$ & $616.1 / 344$ & $815 \pm 195$ & $-569 \pm 4$ & $24 \pm 2$ & $0.54 \pm 0.04$ \\
$\mathrm{~A} / 2$ & $604.0 / 344$ & $806 \pm 59$ & $-1898 \pm 1$ & $29 \pm 1$ & $0.93 \pm 0.03$ \\
$\mathrm{~B} / 2$ & $596.9 / 332$ & $795 \pm 233$ & $-1898 \pm 3$ & $30 \pm 5$ & $0.93 \pm 0.03$ \\
$\mathrm{C} / 2$ & $613.1 / 344$ & $750 \pm 55$ & $-1898 \pm 1$ & $29 \pm 1$ & $0.95 \pm 0.03$ \\
$\mathrm{D} / 2$ & $747.5 / 344$ & $680 \pm 58$ & $-1898 \pm 1$ & $29 \pm 1$ & $0.97 \pm 0.04$ \\
$\mathrm{E} / 2$ & $622.1 / 344$ & $1020 \pm 96$ & $-1895 \pm 1$ & $27 \pm 1$ & $0.90 \pm 0.02$ \\
$\mathrm{~F} / 2$ & $616.1 / 344$ & $993 \pm 98$ & $-1895 \pm 1$ & $27 \pm 1$ & $0.90 \pm 0.02$ \\
\hline
\end{tabular}

a Velocity is relative to a systemic redshift of $c z=4916 \mathrm{~km} \mathrm{~s}^{-1}$ (de Vaucouleurs et al. 1991).

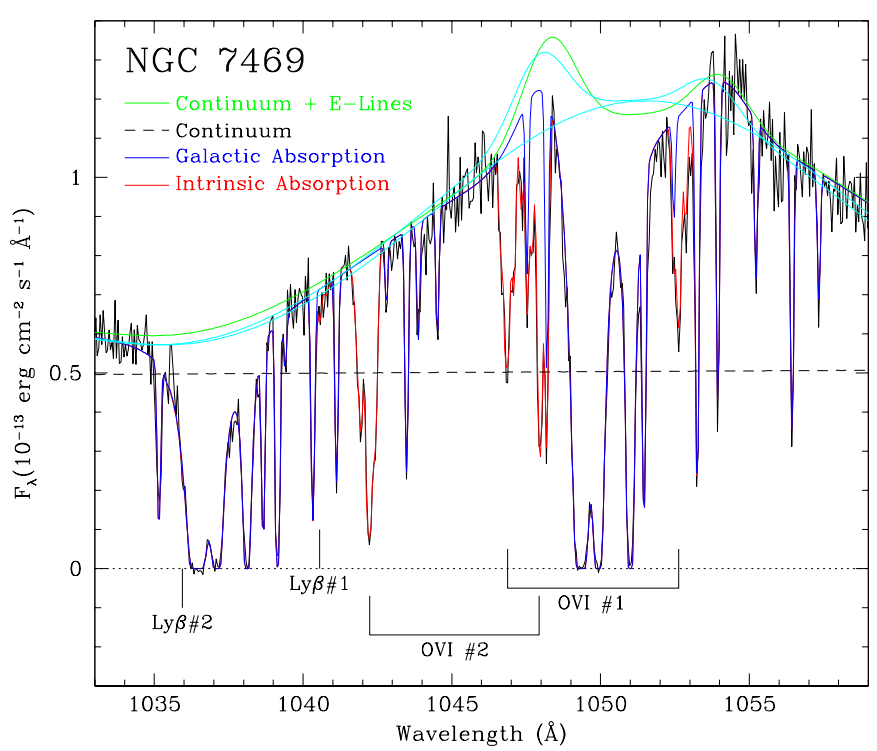

Fig. 2. FUSE spectrum of NGC 7469 (with $0.05 \AA$ bins) in the $\mathrm{Ly} \beta / \mathrm{O}$ VI region is shown as the thin black line. The overlayed color lines show the best fit (model A) as described in the text. The thin green line represents the fitted continuum and emission components. The thin red line shows the fitted intrinsic absorption components. The thin blue line shows the foreground Galactic absorption lines. The light blue lines illustrate the continuum and narrow O VI line profiles for the two extreme cases we considered, models $\mathrm{D}$ and $\mathrm{E}$, which have no narrow line emission, or 2:1 narrow-line emission that is not absorbed, respectively. The intrinsic absorbing gas is readily visible as two separate complexes outlined in red. Corresponding absorption in $\operatorname{Ly} \beta$ is weak at best.

surrounding the two absorption line complexes in O VI and Ly $\beta$ in Fig. 3. Since the relative oscillator strengths of the blue and red lines in the O VI doublet have roughly a 2:1 ratio, one would expect the red line to have a depth that is half that of the blue line in optically thin gas, and an equal depth in optically thick gas. From the normalized spectrum it is readily apparent that the equal depths of the red and blue lines in Component \#1 imply that the absorption is heavily saturated. However, since the

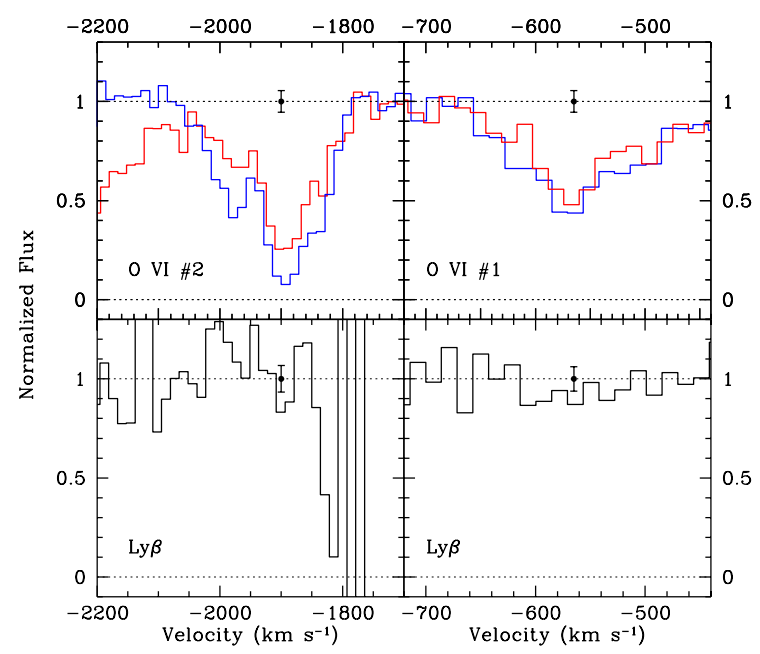

Fig. 3. The normalized line profiles in the upper panels show intrinsic O VI $\lambda 1032$ (the solid blue line) and O VI $\lambda 1038$ (the solid red line) absorbing components 1 and 2. These data are binned at $0.05 \AA$, and foreground Galactic absorption features have been divided out. The normalized profiles in the lower panels show the corresponding location in velocity space of expected $\operatorname{Ly} \beta$ absorption, which is not definitively detected. Representative error bars at the velocity centroid of each component are shown.

line cores are not black at their centers, we also can see that the absorbing gas does not fully cover the underlying emission. The covering fraction is only $\sim 50 \%$. Our fits may underestimate the total column density in this absorption complex. The lack of visible damping wings on the line profile permits us to set an upper limit of $1.5 \times 10^{18} \mathrm{~cm}^{-2}$ on the $\mathrm{O}$ VI column of this component. This upper limit is reflected in the error bar that we assign to the strongest line in Component \#1 in Table 2. The relative depths of the red and blue lines in Component \#2 are not quite equal, but they also are not at an optically thin ratio. This component is approaching saturation, but is not yet optically thick, and so its total column density is better determined. 


\section{Discussion}

As typical for Seyfert 1 galaxies viewed at high spectral resolution in the far-ultraviolet, we see complex, multiple absorption lines from highly ionized O VI. Although Ly $\beta$ absorption in the FUSE spectrum is weak or absent, there are Ly $\alpha$ absorption lines in the FOS spectrum of NGC 7469 obtained in 1996 at systemic velocities of $-1870 \pm 17 \mathrm{~km} \mathrm{~s}^{-1}$ and $-656 \pm 24 \mathrm{~km} \mathrm{~s}^{-1}$ (Kriss et al. 2000a), very close to the velocities of Components \#1 and \#2, so there is some minimal neutral hydrogen column. The high $\mathrm{O}$ VI to H I column-density ratios for both components imply very highly ionized gas consistent with those typical of X-ray warm absorbers and similar to the highest ionization component in Mrk 509 (Kriss et al. 2000b). However, only Component \#1 has velocities comparable to those of higher ionization states of $\mathrm{O}$ VII and $\mathrm{O}$ VIII $\left(-900 \pm 100 \mathrm{~km} \mathrm{~s}^{-1}\right)$ as seen in the XMM-Newton grating spectrum of NGC 7469 (Blustin et al. 2003). The upper limit on the $\mathrm{O}$ VI column density measured in the XMM-Newton grating spectrum of $<10^{16.5} \mathrm{~cm}^{-2}$ is consistent with the column densities measured in the FUSE spectrum.

To test whether the same gas is responsible for both the UV and the X-ray absorption in component \#1, we computed photoionization models similar to those used by Krolik \& Kriss (1995, 2001). These models cover a grid in total column density ranging from $N_{\text {tot }}=10^{18}$ to $10^{21} \mathrm{~cm}^{-2}$, and in ionization parameter from $U=0.05$ to 10.0 , where $U$ is the ratio of ionizing photons in the Lyman continuum to the local electron density. For the illuminating spectrum in our models, we used two different spectral energy distributions. The first, SED1, is the same as that described by Kriss et al. (2000a), which is based on the simultaneous IUE, FOS, and RXTE observations of NGC 7469 performed in 1996. We show SED1 as a solid line in Fig. 4. The second spectral energy distribution, SED2, is based on the current FUSE spectrum presented in this paper and the XMM-Newton spectrum from Blustin et al. (2003). At long wavelengths we assume $f_{v} \propto v^{-1}$, as in SED1. At $2500 \AA$, this breaks to $f_{v} \propto v^{-0.75}$ to match the FUSE spectrum. At X-ray energies, we use the energy index of 0.7 from the XMM-Newton spectrum to span the $0.5 \mathrm{keV}$ to $100 \mathrm{keV}$. At higher energies we use a steeper index of 2.0 to prevent divergence in the total ionizing flux. From $50 \mathrm{eV}$ to $0.5 \mathrm{keV}$, the steep $f_{v} \propto v^{-2.32}$ is a reasonable match to the soft excess seen in the XMM-Newton data. The FUSE and XMM-Newton data were not obtained simultaneously, and so we normalize the X-ray continuum relative to the UV continuum by choosing $\alpha_{\text {ox }}=1.34$, the same value used by Kriss et al. (2000a) based on the 1996 simultaneous observations. SED2 is the dashed line shown in Fig. 4. For comparison, we show the absorptioncorrected UV and X-ray fluxes at $2120 \AA$ and $2 \mathrm{keV}$, respectively, as observed with XMM-Newton (Blustin et al. 2003). These data have $\alpha_{\mathrm{ox}}=1.33 \pm 0.02$, and they are consistent with SED2.

After examining the results of our modeling, we find that none of the models using SED1 achieves sufficiently high ratios of O VI, O VII, and O VIII relative to $\mathrm{H}$ I to match the values observed in our spectra. For SED2, we do find a solution that is compatible with our observations. This model has $U=6.0$

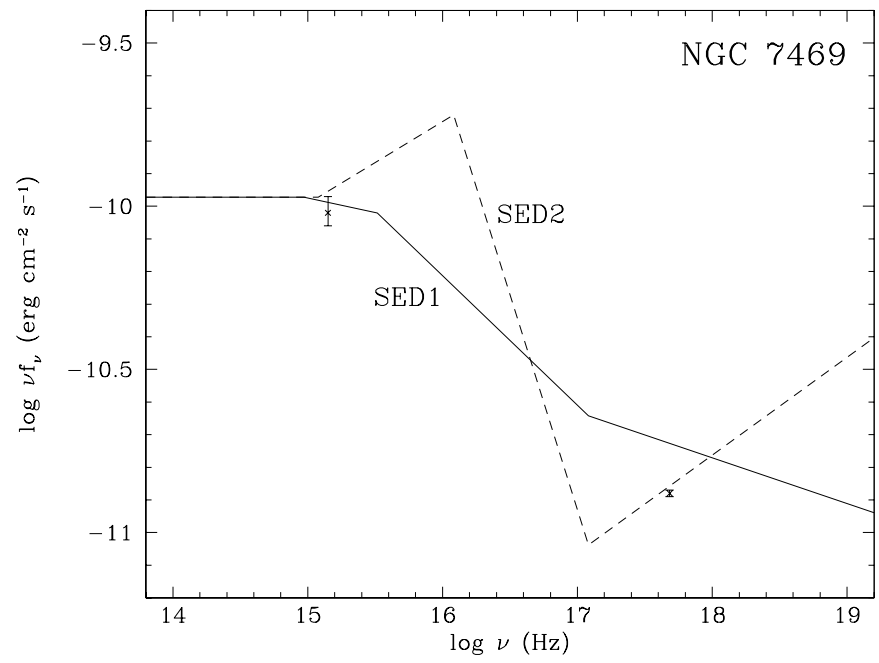

Fig. 4. Spectral energy distributions used for photoionization models of absorbing gas in NGC 7469. SED1, based on Kriss et al. (2000), is the solid line. SED2, based on the spectrum in this paper, is the dashed line. The curves are normalized so that SED2 matches the extinctioncorrected flux at $1000 \AA$ in the FUSE spectrum. The points with error bars showing the UV and X-ray fluxes as observed with XMM-Newton are consistent with SED2.

and $\log N_{\text {tot }}=20.55$. The predicted column densities for ions of interest are summarized in Table 4, where we compare them to the observed column densities. Note that C IV and $\mathrm{N} V$ are virtually invisible in this model, and, indeed, no absorption from these ions was seen by Kriss et al. (2000a) in the 1996 FOS spectrum at the velocity of component \#1.

For Component \#2, the required column density and ionization level is substantially lower. We obtain a reasonable match to the observed $\mathrm{O}$ VI and $\mathrm{H}$ I column densities for $U=0.2$ and $N_{\text {tot }}=18.58$, as also shown in Table 4 . The predicted column densities for $\mathrm{CIV}$ and $\mathrm{N} \mathrm{V}$ in this model are also rather low, although easily detectable. We note that the UV continuum in the current FUSE observation is a factor of $2.1 \times$ brighter than that observed with the FOS in 1996. If we assume that any variations since then are simply due to changes in flux with a constant column density of absorbing gas, then we would have expected the gas in 1996 to be responding to an ionization parameter of $U=0.08$. This would then predict $\mathrm{C}$ IV and $\mathrm{N} \mathrm{V}$ column densities of $5.7 \times 10^{13} \mathrm{~cm}^{-2}$ and $7.2 \times 10^{13} \mathrm{~cm}^{-2}$, respectively, with corresponding equivalent widths of the blue components of each doublet (for optically thin gas) of $0.2 \AA$ and $0.16 \AA$. While these are still lower than the observed values in the FOS spectrum, $(0.45 \pm 0.05$ and $0.48 \pm 0.08 \AA$ for $\mathrm{C}$ IV and $\mathrm{N} \mathrm{V}$, respectively) given the uncertainties in the spectral energy distribution and other potentially variable factors, we find the agreement quite good.

The covering fraction and line depth of Component \#1 is consistent with its absorbing only the continuum flux and none of the emission-line flux in NGC 7469. Its high ionization parameter, and its likely identification with the same gas responsible for the X-ray absorption suggests that it is much higher ionization than Component \#2. Taken together, these points imply that Component \#1 lies very close to the central engine, 
Table 4. Photoionization models for the absorbers in NGC 7469.

\begin{tabular}{cccccccc}
\hline \hline$U$ & $\begin{array}{c}\log N_{\text {tot }} \\
\left(\mathrm{cm}^{-2}\right)\end{array}$ & $\begin{array}{c}N(\mathrm{HI}) \\
\left(\mathrm{cm}^{-2}\right)\end{array}$ & $\begin{array}{c}N(\mathrm{O} \mathrm{VI}) \\
\left(\mathrm{cm}^{-2}\right)\end{array}$ & $\begin{array}{c}N(\mathrm{O} \text { VII }) \\
\left(\mathrm{cm}^{-2}\right)\end{array}$ & $N(\mathrm{O}$ VIII $)$ & $N(\mathrm{C} \mathrm{IV})$ & $N(\mathrm{~N} \mathrm{~V})$ \\
\hline $6.0^{\mathrm{a}}$ & 20.55 & $1.1 \times 10^{14}$ & $8.0 \times 10^{14}$ & $7.3 \times 10^{16}$ & $1.3 \times 10^{17}$ & $1.1 \times 10^{12}$ & $1.0 \times 10^{13}$ \\
& Observed: & $(8.0 \pm 4.2) \times 10^{14}$ & $(8.0 \pm 3.0) \times 10^{14}$ & $\left(3_{-3}^{+5}\right) \times 10^{16}$ & $\left(2_{-1}^{+2}\right) \times 10^{17}$ & $\ldots$ & $\ldots$ \\
$0.2^{\mathrm{b}}$ & 18.58 & $6.9 \times 10^{13}$ & $5.5 \times 10^{14}$ & $8.2 \times 10^{14}$ & $2.0 \times 10^{13}$ & $2.4 \times 10^{13}$ & $4.3 \times 10^{13}$ \\
& Observed: & $(2.1 \pm 4.8) \times 10^{13}$ & $(8.0 \pm 2.3) \times 10^{14}$ & $<3 \times 10^{16}$ & $<3 \times 10^{16}$ & $\ldots$ & $\ldots$ \\
$0.08^{\mathrm{c}}$ & 18.58 & $2.0 \times 10^{14}$ & $6.7 \times 10^{14}$ & $8.7 \times 10^{14}$ & $2.9 \times 10^{12}$ & $5.7 \times 10^{13 \mathrm{~d}}$ & $7.2 \times 10^{14 \mathrm{e}}$ \\
\hline
\end{tabular}

a This model is our best match to Component \#1.

b This model is our best match to Component \#2.

${ }^{c}$ This model corresponds to Component \#2 at the epoch of the 1996 FOS observations.

${ }^{\mathrm{d}}$ For a Doppler width of $100 \mathrm{~km} \mathrm{~s}^{-1}$, the blue line in the doublet would have an equivalent width of $0.20 \AA$.

${ }^{\mathrm{e}}$ For a Doppler width of $100 \mathrm{~km} \mathrm{~s}^{-1}$, the blue line in the doublet would have an equivalent width of $0.16 \AA$.

possible interior to the broad emission line region. This would favor its association with an accretion disk wind, rather than a thermally driven wind emanating from the obscuring torus. In constrast, the high covering fraction of Component \#2 clearly places it exterior to both the BLR and continuum regions as would be expected for the thermally driven winds of Krolik \& Kriss $(1995,2001)$. Overall, in this one object we may be seeing absorption from both a torus wind and a disk wind.

\section{Summary}

Our high-resolution far-ultraviolet spectrum of NGC 7469 obtained with FUSE shows broad emission lines of C III, $\mathrm{N}$ III, O VI, and $\mathrm{He}$ II, as well as possible emission from $S$ IV $\lambda \lambda 1062,1072$. Intrinsic absorption in the $\mathrm{O}$ VI $\lambda \lambda 1032,1038$ resonance doublet arises in two distinct kinematic components at systemic velocities of $-569 \mathrm{~km} \mathrm{~s}^{-1}$ (Component \#1) and $-1898 \mathrm{~km} \mathrm{~s}^{-1}$ (Component \#2). Both components are very highly ionized with no significant $\mathrm{Ly} \beta$ absorption detected at either velocity. Component \#2, although highly ionized, has a lower total column density than Component \#1, and it is consistent with having no associated X-ray absorption. It covers more than $90 \%$ of both the continuum and broad-line emission. Component \#1 at $-569 \mathrm{~km} \mathrm{~s}^{-1}$ is the best match in velocity to the highly ionized X-ray absorbing gas detected in the XMM-Newton grating spectrum of NGC 7469 (Blustin et al. 2003) at a blueshift of $900 \pm$ $100 \mathrm{~km} \mathrm{~s}^{-1}$. Photoionization models for Component \#1 show that for a total column density of $10^{20.55} \mathrm{~cm}^{-2}$ and an ionization parameter of $U=6.0$, the column densities of $\mathrm{H} \mathrm{I}, \mathrm{O}$ VI, $\mathrm{O}$ VII, and O VIII in the FUSE and the XMM-Newton spectra can all be reproduced. Component \#1 also has an extraordinarily low covering fraction of 0.5 , and is consistent with covering only the continuum emission and none of the broad-line emission. This suggests that it might arise in an accretion disk wind interior to the broad line region.

Acknowledgements. This work is based on data obtained for the Guaranteed Time Team by the NASA-CNES-CSA FUSE mission operated by the Johns Hopkins University. Financial support to U. S. participants has been provided by NASA contract NAS5-32985. The U.K. authors acknowledge the support of the Particle Physics and Astronomy Research Council. G. Kriss acknowledges additional support from NASA Long Term Space Astrophysics grant NAGW-4443.

\section{References}

Blustin, A., Branduardi-Raymont, G., Behar, E., et al. 2003, A\&A, 403, 481

Cardelli, J., Clayton, G., \& Mathis, J. 1989, ApJ, 345, 245

Collier, S., Horne, K., Kaspi, S., et al. 1998, ApJ, 500, 162

Crenshaw, D. M., Kraemer, S. B., Boggess, A., et al. 1999, ApJ, 516, 750

Crenshaw, D. M., Maran, S. P., \& Mushotzky, R. F. 1998, ApJ, 496, 797

de Vaucouleurs, G., de Vaucouleurs, A., Corwin, H. G., et al. 1991, Third Reference Catalogue of Bright Galaxies (New York: Springer)

Espey, B. R., Kriss, G. A., Krolik, J. H., et al. 1998, ApJ, 500, L13

George, I. M., Turner, T. J., Netzer, H., et al. 1998, ApJS, 114, 73

Hamann, F., Barlow, T. A., Beaver, E. A., Burbidge, E. M., \& Cohen, R. D. 1995, ApJ, 443, 606

Hamann, F., Barlow, T. A., \& Junkkarinin, V. 1997, ApJ, 478, 87

Kaastra, J. S., Mewe, R., Liedahl, D. A., Komossa, S., \& Brinkman, A. C. 2000, A\&A, 354, L83

Kaspi, S., Brandt, W. N., Netzer, H., et al. 2000, ApJ, 535, L17

Kaspi, S., Brandt, W. N., George, I. M., et al. 2002, ApJ, 574, 643

Königl, A., \& Kartje, J. F. 1994, ApJ, 434, 446

Kriss, G. A. 1994, in Astronomical Data Analysis Software and Systems III, ed. D. R. Crabtree, R. J. Hanisch, \& J. Barnes (San Francisco: ASP), ASP Conf. Ser., 61, 437

Kriss, G. A., Peterson, B. M., Crenshaw, D. M., \& Zheng, W. 2000a, ApJ, 535, 58

Kriss, G. A., Green, R. F., Brotherton, M., et al. 2000b, ApJ, 538, L17

Kriss, G. A., Green, R. F., Brotherton, M., et al. 2003, ApJS, to be submitted

Krolik, J. H., \& Kriss, G. A. 1995, ApJ, 447, 512

Krolik, J. H., \& Kriss, G. A. 2001, ApJ, 561, 684

Laor, A., Bahcall, J. N., Jannuzi, B. T., Schneider, D. P., \& Green, R. F. 1995, ApJS, 99, 1

Mathur, S., Elvis, M., \& Wilkes, B. 1999, ApJ, 519, 605

Moos, H. W., Cash, W. C., Cowie, L. L., et al. 2000, ApJ, 538, L1

Murray, N., Chiang, J., Grossman, S. A., \& Voigt, G. M. 1995, ApJ, 451,498

Nandra, K., Clavel, J., Edelson, R. A., et al. 1998, ApJ, 505, 594

Netzer, H., Chelouche, D., George, I. M., et al. 2002, ApJ, 538, L1

Reynolds, C. S. 1997, MNRAS, 286, 513

Sahnow, D. J., Moos, H. W., Ake, T. B., et al. 2000, ApJ, 538, L7

Savage, B. D., \& Sembach, K. S. 1996, ARA\&A, 34, 279

Schlegel, D. J., Finkbeiner, D. P., \& Davis, M. 1998, ApJ, 500, 525

Wanders, I., Peterson, B. M., Alloin, D., et al. 1997, ApJS, 113, 69 